\title{
A Fully Automatic Deep Learning Method for Atrial Scarring Segmentation from Late Gadolinium-Enhanced MRI Images
}

\author{
Guang Yang ${ }^{* 1,2}$, Xiahai Zhuang ${ }^{* 3}$, Habib Khan ${ }^{1,2}$, Shouvik Haldar ${ }^{1}$, Eva Nyktari ${ }^{1}$, \\ Xujiong Ye ${ }^{4}$, Greg Slabaugh ${ }^{5}$, Tom Wong ${ }^{1}$, Raad Mohiaddin ${ }^{1,2}$, Jennifer Keegan ${ }^{1,2}$, David Firmin ${ }^{1,2}$ \\ ${ }^{1}$ Cardiovascular Biomedical Research Unit, Royal Brompton Hospital, SW3 6NP, London, UK \\ ${ }^{2}$ National Heart and Lung Institute, Imperial College London, SW7 2AZ, London, UK \\ ${ }^{3}$ School of Data Science, Fudan University, Shanghai, 200000, China \\ ${ }^{4}$ School of Computer Science, University of Lincoln, LN6 7TS, Lincoln, UK \\ ${ }^{5}$ Department of Computer Science, City University London, EC1V 0HB, London, UK
}

\begin{abstract}
Precise and objective segmentation of atrial scarring (SAS) is a prerequisite for quantitative assessment of atrial fibrillation using non-invasive late gadolinium-enhanced (LGE) MRI. This also requires accurate delineation of the left atrium (LA) and pulmonary veins (PVs) geometry. Most previous studies have relied on manual segmentation of LA wall and PVs, which is a tedious and error-prone procedure with limited reproducibility. There are many attempts on automatic SAS using simple thresholding, histogram analysis, clustering and graph-cut based approaches; however, in general, these methods are considered as unsupervised learning thus subject to limited segmentation accuracy. In this study, we present a fully-automated multi-atlas based whole heart segmentation method to derive the LA and PVs geometry objectively that is followed by a fully automatic deep learning method for SAS. Our deep learning method consists of a feature extraction step via super-pixel over-segmentation and a supervised classification step via stacked sparse auto-encoders. We demonstrate the efficacy of our method on 20 clinical LGE MRI scans acquired from a longstanding persistent atrial fibrillation cohort. Both quantitative and qualitative results show that our fully automatic method obtained accurate segmentation results compared to the manual segmentation based ground truths.
\end{abstract}

\section{INTRODUCTION}

Atrial fibrillation $(\mathrm{AF})$ is the most common sustained heart rhythm disturbance encountered in adult cardiology. Several studies have shown that $\mathrm{AF}$ is correlated with electrical, contractile, and structural remodeling in the left atrium (LA) [1]. Moreover, LA fibrosis may be arrhythmogenic that causes more aggressive symptoms and makes difficulties in the management of $\mathrm{AF}$ [1]. Minimally invasive catheter ablation (CA) using radio-frequency energy has become one of the most common treatments for $\mathrm{AF}$ patients refractory to drug treatment [2]. CA aims to electrically isolate the pulmonary veins (PVs) from the left atrial (LA) body because previous studies show that ectopic beats from the PVs can frequently trigger the AF [3]. In this context, techniques have been developed to evaluate the LA wall composition and assess the circumferential PVs scarring that results from CA in order to understand the $\mathrm{AF}$ with proper management and prognosis. At present, electro-anatomical mapping (EAM) system, which is performed during the electrophysiological study, is considered to be a clinical reference standard technique for the assessment of the LA substrate and ablation-induced scarring. However, EAM is invasive and suffers from ionizing radiation and its suboptimal accuracy, which has reported errors of up to $10 \mathrm{~mm}$ in the localization of scar tissue [4].

Supported by British Heart Foundation Project Grant (Project Number: PG/16/78/32402) and NIHR. Asterisk indicates corresponding authors. Emails: GY—g.yang@imperial.ac.uk and XZ—zhuangxiahai@163.com.
Noninvasive late gadolinium-enhanced (LGE) MRI is an established method for visualizing and assessing myocardial infarction or fibrosis [5]. This is ascribed to the altered wash-in and wash-out contrast agent kinetics, and the hyper-enhancement reflects the increased interstitial space of the myocardium with fibrosis while healthy myocardium is 'nulled'. In addition, the successful imaging of atrial scarring has been demonstrated using LGE MRI [6]. However, clinical interpretation of these tomographic LGE MRI scans for AF patients is difficult because: (1) residual respiratory motion, heart rate variability, low signal-to-noise ratio (SNR), and contrast agent wash-out during the long acquisition (current scanning time 10mins) frequently result in image quality being poor and (2) the thin LA wall with surrounding structures such as blood, aorta, spine, and esophagus may limit the correct selection of the LA myocardium on LGE MRI images and subsequently result in a large number of false positives for the atrial scarring delineation. Essentially, precise and objective assessment of atrial scarring requires two segmentations: (1) the delineation of LA and PVs geometry and (2) the segmentation of atrial scarring (SAS).

For the LA and PVs geometry, most previous studies have relied on manual delineation [2], [7]-[10]. Knowles et al. [11] used a semi-automatic thresholding and region growing method to extract the LA and PVs anatomy. More recently, Karim et al. [12] utilized a statistical shape model to solve the LA and PVs geometry but subject to manual corrections. In [13], an automatic atlas based method has been applied; however, local level set based refinement is required using co-registered MR angiography (MRA) data. MRA data are generally acquired in an inspiratory breath-hold and without cardiac gating; therefore, the anatomy extracted from MRA can be highly deformed compared to that acquired by LGE MRI and this may cause difficulties in the co-registration step and subsequently mistake the SAS.

Several strategies have been proposed for visualization of the atrial scarring, e.g., maximum intensity projection (MIP) [6], [11], [13], [14]. MIP based methods can provide more intuitive visualization and may have a potential role for guiding CA procedures. However, the major drawback of this technique is that it is only a visualization of the hyper-enhancement, but not a segmentation method that can result in volumetric quantification [12]. Oakes et al. [7] segmented the enhanced atrial scarring by analyzing the intensity histogram of the manually delineated LA wall. Perry et al. [8] applied k-means clustering to quantitatively assess normal and scarred tissue from manual defined LA and PVs geometry. Karim et al. [12] investigated a graph-cut based segmentation method to differentiate atrial scarring from healthy myocardium. A grand challenge was carried out that attracted 8 research groups who submitted their atrial segmentation algorithms including histogram analysis, simple and advanced thresholding, 
k-means clustering, and graph-cuts methods to establish a benchmarking work [9]. The benchmarking work was tested on data acquired from multiple institutions, and the LA endocardium and cavity for each scan was also provided to all the participants. The best performing algorithms in the benchmarking work have shown promising results, but there are large variances in the performance especially for the pre-ablation datasets where image quality is generally worse and native scarring is more diffuse. Due to the aforementioned limitations, i.e., poor image quality and thinner LA wall with confounded adjacent structures, the precise and objective assessment of atrial scarring is still a very challenging problem. And the subjective and inaccurate segmentation could be one of the major reasons that the correlation between atrial scarring identified by LGE MRI (enhanced regions) and EAM (low voltage regions) has been questioned [15].

In this study, we present a fully automatic framework that yields an efficient and objective atrial scarring assessment including: (1) a fully-automated multi-atlas based whole heart segmentation (MA-WHS) method to solve the LA and PVs anatomy and (2) a fully automatic deep learning method for SAS. Our deep learning method consists of a feature extraction step via super-pixel over-segmentation and a supervised classification step via stacked sparse auto-encoders (SSAE). Compared with the ground truth formed by manual delineation, our fully automatic method obtains promising segmentation results, which are also comparable to the state-of-the-art SAS methods.

\section{METHODS}

The overall workflow of our method is summarized in Figure 1, and details of each step are described below.

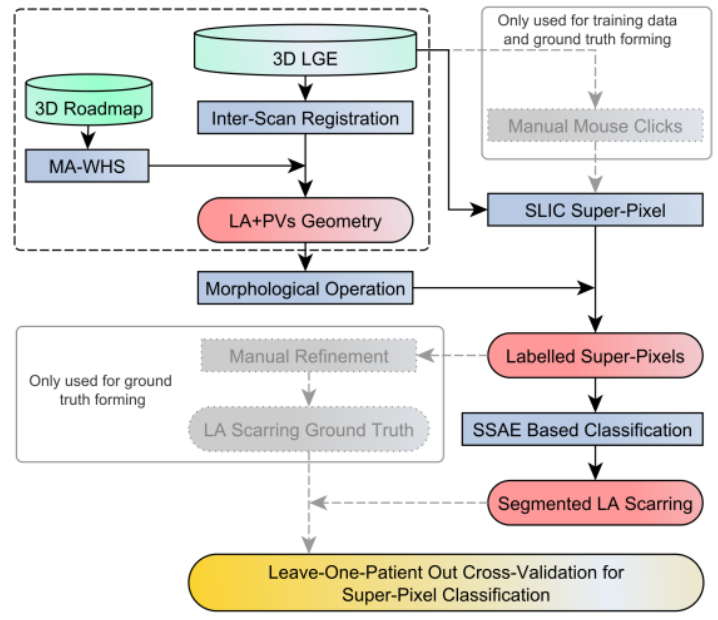

Figure 1: Flowchart of our fully automatic framework.

\subsection{Patients and Data Acquisition}

In agreement with the local regional ethics committee, cardiac MRI was performed in longstanding persistent AF patients between 2011-2013. The image quality of each dataset was scored by a senior cardiac MRI physicist on a Likert-type scale-0 (non-diagnostic), 1 (poor), 2 (fair), 3 (good) and 4 (very good) - depending on the level of SNR, appropriate inversion time, and the existence of navigator beam and ghost artifacts. Ten pre-ablation scans with image quality greater or equal to 2 have been retrospectively entered into this study ( $\sim 60 \%$ of all the scanned pre-ablation cases). To make a balanced dataset, we randomly selected 10 post-ablation cases from all the 26 post-ablation scans with image quality greater or equal to $2(\sim 92 \%$ of all the scanned post-ablation cases).
Cardiac MR data were acquired on a Siemens Magnetom Avanto 1.5T scanner. Transverse navigator-gated 3D LGE MRI [6], [7], [16] was performed using an inversion prepared segmented gradient echo sequence (TE/TR $2.2 \mathrm{~ms} / 5.2 \mathrm{~ms}$ ) 15 minutes after gadolinium $(\mathrm{Gd})$ administration when a transient steady-state of Gd wash-in and wash-out of normal myocardium had been reached [17]. LGE MRI images were scanned with a field-of-view $380 \times 380 \mathrm{~mm}^{2}$ and reconstructed to $60-68$ slices at $0.75 \times 0.75 \times 2 \mathrm{~mm}^{3}$.

The LA and PVs geometry would ideally be segmented directly using 3D LGE MRI dataset. However, this is difficult because healthy myocardium is 'nulled' and only scar tissue is seen with high signal. In this study, instead of using un-gated MRA, a respiratory and cardiac gated 3D Roadmap image, i.e., using a balanced steady state free precession sequence (TE/TR $1 \mathrm{~ms} / 2.3 \mathrm{~ms}$ ), has been acquired for each patient to resolve the cardiac anatomy. The Roadmap data were acquired with a field-of-view $380 \times 380 \mathrm{~mm}^{2}$ and reconstructed to 160 slices at $0.8 \times 0.8 \times 1.6 \mathrm{~mm}^{3}$. Both 3D LGE MRI and Roadmap data were acquired during free-breathing using a crossed-pairs navigator positioned over the dome of the right hemi-diaphragm with navigator acceptance window size of $5 \mathrm{~mm}$ and CLAWS respiratory motion control [18].

\subsection{Multi-Atlas Based Whole Heart Segmentation}

A MA-WHS method was developed to derive the LA and PVs (LA+PVs) geometry [19], [20]. It was applied on the Roadmap images and then mapped to LGE MRI (dashed box region in Figure 1). This segmentation consists of two major steps: (1) atlas propagation based on image registration algorithms and (2) label fusion from multi-atlas propagated segmentation results.

The whole heart atlases were constructed using 30 MRI Roadmap studies retrieved from the Left Atrium Segmentation Grand Challenge organized by King's College London [21]. For each atlas dataset, we have manual labels of the right and left ventricles, the right and left atria, the aorta, the pulmonary artery, the pulmonary veins and the appendages. MA-WHS executes an atlas-to-target registration in order to maximizing the similarity between the target image ( $I)$ and each atlas dataset $\left(\boldsymbol{A}_{a}, \boldsymbol{L}_{a} \mid a=1, \ldots N\right)$, where $\boldsymbol{A}_{a}$ and $\boldsymbol{L}_{a}$ are the intensity image and the corresponding segmentation label image of the $a$-th atlas $(N=30)$. Then a set of warped atlases can be derived $\left\{\left(A_{a}, L_{a}\right) \mid a=1, \ldots N\right\}$, where $A_{a}$ and $L_{a}$ are the warped atlas intensity image and corresponding segmentation result. In this study, a hierarchical registration scheme was applied for segmentation propagation [22].

In order to generate one final segmentation for the LA+PVs geometry from the 30 propagated results, a label fusion algorithm is required. This can be achieved using local weighted label fusion, which evaluates local similarity between patches from the atlases and the target image, that is

$$
L_{I}(x)=\underset{l \in\left\{l_{b k}, l_{l a}\right\}}{\operatorname{argmax}} \sum_{a} w_{a}\left(S\left(I, A_{a}, x\right)\right) \delta\left(L_{a}(x), l\right)
$$

where $l_{b k}$ and $l_{l a}$ are the labels of the background and LA+PVs, respectively, and the local weight $w_{a}(\cdot) \propto S(\cdot)$ is determined by the local similarity $S(\cdot)$ between the target image and the atlas. $\delta(p, q)$ is the Kronecker delta function which returns 1 when $p=q$ and returns 0 otherwise. In this study, we extended this to a multi-scale patch based label fusion (MSP-LF) [23], and the patches computed from different scale spaces can represent the different levels of structural information, with low scale capturing local fine structure and high scale suppressing fine structure but providing global structural information of the image. In addition, we adopted the multi-resolution implementation and couple it with the MSP where the high-scale patches can be efficiently computed using a 
low-resolution image space. The local similarity between two images using the MSP measure is computed as

$$
S_{\mathrm{msp}}\left(I, A_{a}, x\right)=\sum_{s} S\left(I^{(s)}, A_{a}^{(s)}, x\right)
$$

in which $I^{(s)}=I * \operatorname{Gaussian}\left(0, \sigma_{s}\right)$ is the target image from $s$ scale-space that is computed from the convolution of the target image with Gaussian kernel function with scale $s$. Here, we computed the local similarity in multi-scale image using the conditional probability of the images, that is

$$
S\left(I^{(s)}, A_{a}^{(s)}, x\right)=p\left(i_{x} \mid j_{x}\right)=\frac{p\left(i_{x}, j_{x}\right)}{p\left(j_{x}\right)}
$$

where $i_{x}=I^{(s)}(x)$ and $j_{x}=A_{a}^{(s)}(x)$ and the conditional image probability is obtained from the joint and marginal image probability which can be calculated using the Parzen window estimation [24].

For each patient case, the Roadmap dataset was then registered to the LGE MRI dataset using the DICOM header data, and then refined by affine and nonrigid registration steps [25]. The resulting transformation was applied to the MA-WHS derived cardiac anatomy to define the LA+PVs geometry on the LGE MRI dataset.

\subsection{Feature Extraction-Super-Pixel Based Over-segmentation}

Simple Linear Iterative Clustering (SLIC) [26] was used to separate potential enhanced atrial scarring regions from other healthy myocardium regions after the LA+PVs geometry was determined. Based on local k-means clustering, the SLIC method iteratively groups pixels into perceptually meaningful patches, i.e., super-pixels. In this study, SLIC is initialized by sampling the target slice of the LGE MRI image into a regular grid space with grid interval of 4 pixels (i.e., $3 \times 3 \mathrm{~mm}^{2}$ ) considering the LA wall thickness is approximately $3 \mathrm{~mm}$, and also take into account that the super-pixel size is still large enough to extract statistics of the grouped pixel intensities.

The super-pixel over-segmentation has two uses in this study: (1) it has been used as a by-product tool, which can help cardiologists to construct a manual ground-truth segmentation of the atrial scarring easily and (2) the labeled super-pixels with their intensity features will be used to train a classifier that yields a fully automatic SAS (Figure 1). The workflow can be summarized in the following steps:

(1) We asked experienced cardiologists specialized in cardiac MRI to perform manual mouse clicks on the LGE MRI images to label the enhanced atrial scarring regions. The manual mouse clicks were done on the original LGE MRI images without the super-pixel grid overlaid, which may reduce the visibility of the enhancement on LGE MRI images. The coordinates of the mouse clicks were used to select the enhanced super-pixels. Only one mouse click will be taken into account if multiple clicks dwell in the same super-pixel.

(2) The LA+PVs geometry was determined by our MA-WHS method. We then applied a morphological dilation (assuming LA wall thickness is $3 \mathrm{~mm}$ ) to extract the LA wall and PVs. The blood pool regions were extracted by a morphological erosion $(5 \mathrm{~mm})$ from the endocardial LA boundary and the pixel intensities were normalized according to the blood pool intensities [9].

(3) We masked the selected enhanced super-pixels using the LA wall and PVs segmentation. Only the super-pixels having a defined overlap ( $\geq 20 \%$ ) with the LA wall and PVs segmentation were selected as enhancement. Other super-pixels were discarded as they were considered to be enhancement from other confounded tissues. The other super-pixels overlapped with the LA wall and PVs but not selected as enhancement were considered as non-enhancement. For each super-pixel, eight intensity features were extracted including minimum, maximum, mean, median, standard deviation, kurtosis, mode, and entropy. Together with the super-pixel labels, the training dataset was formed.

(4) Once we extracted the enhanced super-pixels, they were combined to create a binary image for each slice, i.e., 1 for enhanced super-pixels and 0 for unenhanced. The binary image was overlaid on the original LGE MRI images and our cardiologists performed manual corrections to create the final boundaries (ground truths) of the enhanced atrial scarring.

\subsection{Supervised Classification-Stacked Sparse Auto-Encoders}

After the training dataset was established, Stacked Sparse Auto-encoders (SSAE) based classification was applied to classify the labeled super-pixels and subsequently derive the final SAS (Figure 1). SSAE is a type of deep learning architecture, which is built by stacking multiple layers of basic Sparse Auto-encoder (SAE), in which the outputs of each layer is wired to the inputs of the successive layer, and connecting a Softmax classifier as the final layer of the network for accomplishing the classification task (Figure 2). In addition, each $\mathrm{SAE}$ is a multilayer feed-forward neural network trained to represent the input with backpropagation. First, the unsupervised pre-training of such architecture is performed one layer at a time by minimizing the error in reconstructing its input and learning an encoder and a decoder, which yields a set of weights $\mathrm{W}$ and biases b. Second, the Softmax layer is trained in a supervised fashion. Third, supervised fine-tuning is used to improve the deep neural network performance using backpropagation on the whole multilayer network. More details of the SSAE can be found elsewhere [27]-[29].

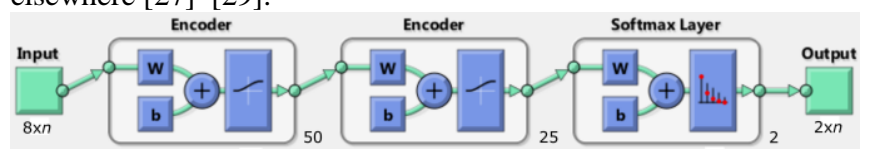

Figure 2: The architecture of SSAE and Softmax classifier for super-pixel classification $(8$ is the number of intensity features, $n$ is the number of super-pixels used for training, 50 and 25 are the sizes of the hidden layers).

\subsection{Experimental Settings and Performance Measure}

In this study, we validated the LA+PVs segmentation and the SAS respectively against established ground truths from manual segmentations by experienced expert cardiologists. For the LA+PVs segmentation, Dice score [30], Hausdorff distance (HD) [31] and Average Surface Distance (ASD) [30] were used as evaluation metrics. For the SAS, we employed leave-one-patient-out cross-validation (LOO CV) and reported the cross-validated accuracy, sensitivity, specificity, area under the receiver operating characteristic (ROC) curve (AUC), and the Dice score [32]-[34].

\section{RESULTS}

Figure 3 shows the training data construction procedure (refer to the subsection 2.3 and Figure 1). We can observe that the labeled super-pixels (enhanced or non-enhanced) are not restricted by the LA wall thickness (i.e., LA wall and PVs regions between the green and magenta curves as shown in Figure 3 (i-j)). Table 1 tabulates the quantitative results of our MA-WHS and final SAS. For MA-WHS, we obtained a mean Dice score of 0.9, mean ASD of $1.5 \mathrm{~mm}$, and mean $\mathrm{HD}$ of $9.5 \mathrm{~mm}$. Most of the main LA volumes were segmented accurately with the majority of errors appearing at distal PVs regions. For SAS, we achieved classification with accuracy of 0.89 and AUC of 0.94. The final Dice score of SAS is $0.78 \pm 0.08$ (Table 1). Compared with standard implementations of other methods, our technique obtained superior SAS (Figure 4). Figure 5 shows the final qualitative comparison results of SAS between the ground truth and our fully-automated super-pixel classification based framework in an example pre-ablation and an example post-ablation study.

\section{DISCUSSION AND CONCLUSION}

In this work, we presented a fully automatic framework to 
differentiate enhanced atrial scarring from LGE MRI images. The framework consists of two major components, i.e., MA-WHS and SAS, which is achieved by SSAE based deep learning for super-pixel classification. Experiments on 20 clinical studies have shown promising results compared to the established ground truth.

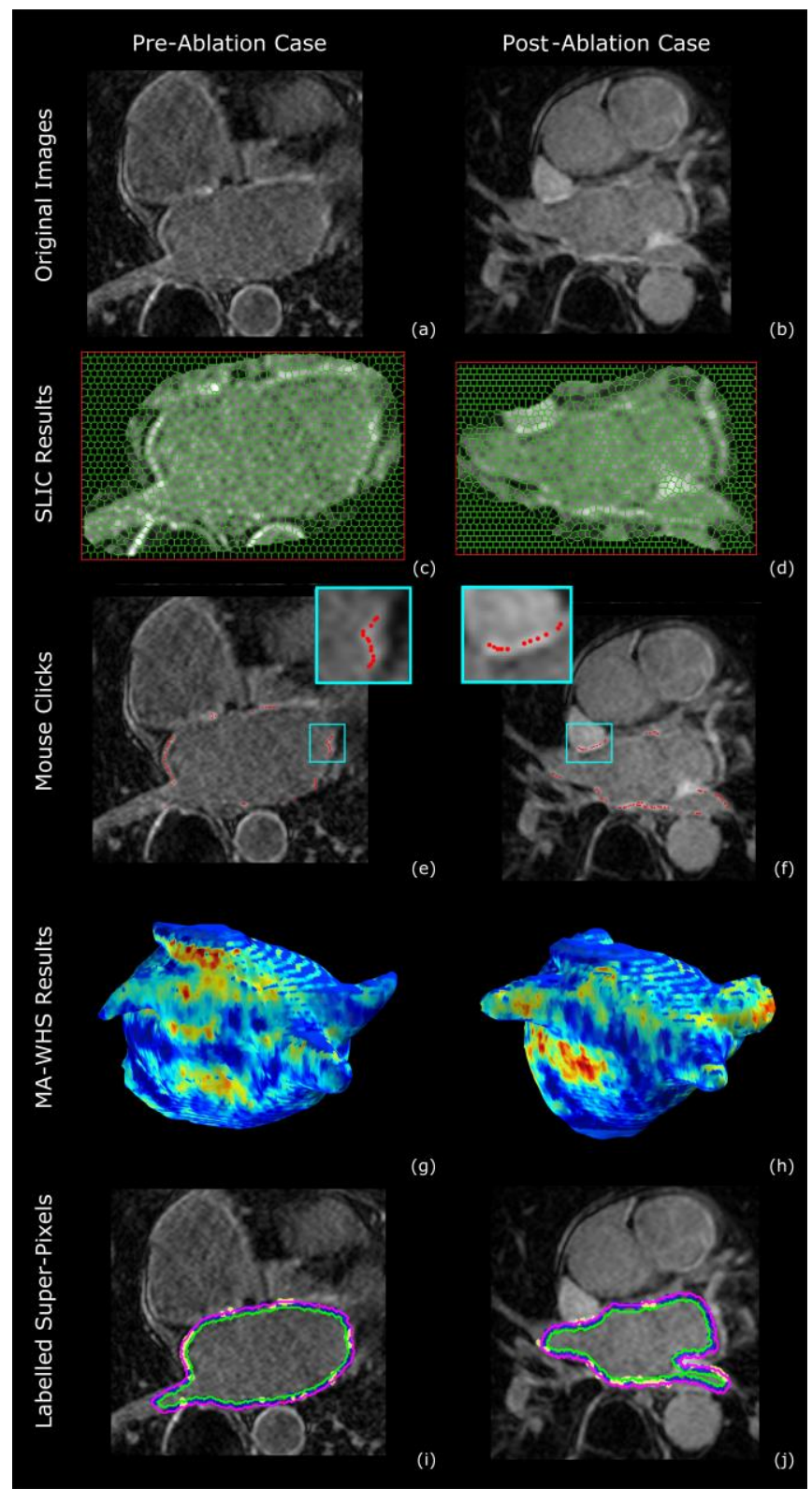

Figure 3: Training data construction for an example pre-ablation (left column) and an example post-ablation (right column) case. (a-b): original images; (c-d): SLIC super-pixels on ROIs of LA+PVs derived from MA-WHS; (e-f): manual mouse clicks; (g-h) MA-WHS results with 3D MIP; (i-j): labeled training dataset for further classification (yellow: labeled enhanced atrial scarring; blue: labeled non-enhanced super-pixels).

Table 1: Quantitative results of MA-WHS and final SAS

\begin{tabular}{cccccccc}
\hline & Accuracy & Sensitivity & Specificity & AUC & Dice Score & HD $(\mathrm{mm})$ & ASD $(\mathbf{m m})$ \\
\hline MA-WHS & - & - & - & - & $0.90 \pm 0.12$ & $9.53 \pm 6.01$ & $1.47 \pm 0.89$ \\
\hline SAS & 0.89 & 0.95 & 0.71 & 0.94 & $0.78 \pm 0.08$ & - & - \\
\hline
\end{tabular}

Compared to existing methods with manually delineated $\mathrm{LA}+\mathrm{PVs}$, our method obtained significantly higher Dice scores (Figure 4). The four methods we compared in this study were described in the benchmarking work [9]; however, we only implemented standard versions of these algorithms and did not perform further optimization. Moreover, the datasets for which those algorithms were tuned are different from those used in our study; therefore, a totally fair comparison may not be possible although similar performance can be observed between our implementation and those reported in [9] especially for the pre-ablation cases. For post-ablation cases, our SAS results showed similar results to the best performed method reported in [9] but with smaller variance, which may be due to the fact that the datasets used in the benchmarking work were acquired from multiple institutions.

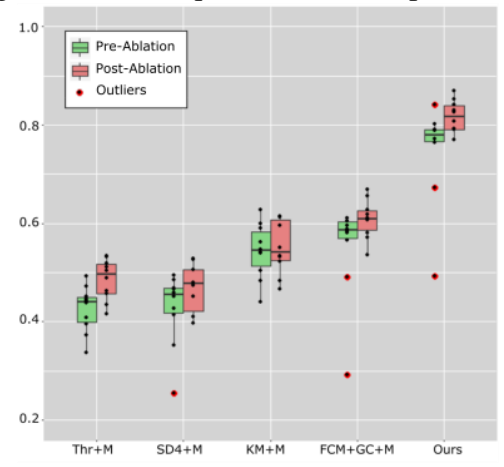

Figure 4: Comparison results with other atrial scarring segmentation methods using DICE. Thr: simple thresholding; SD4: conventional standard deviation (4 SDs were tested); KM: k-means clustering; FCM+GC: fuzzy c-means clustering with graph-cuts; $+\mathrm{M}$ : with manual delineated LA+PVs.

SSAE has been successfully applied for various classification tasks, and it has also proved to be a powerful feature extractor. A possible limitation of our study is that we still used handcrafted intensity features derived from our super-pixels. In the future work, we will investigate the features directly derived from the SSAE.

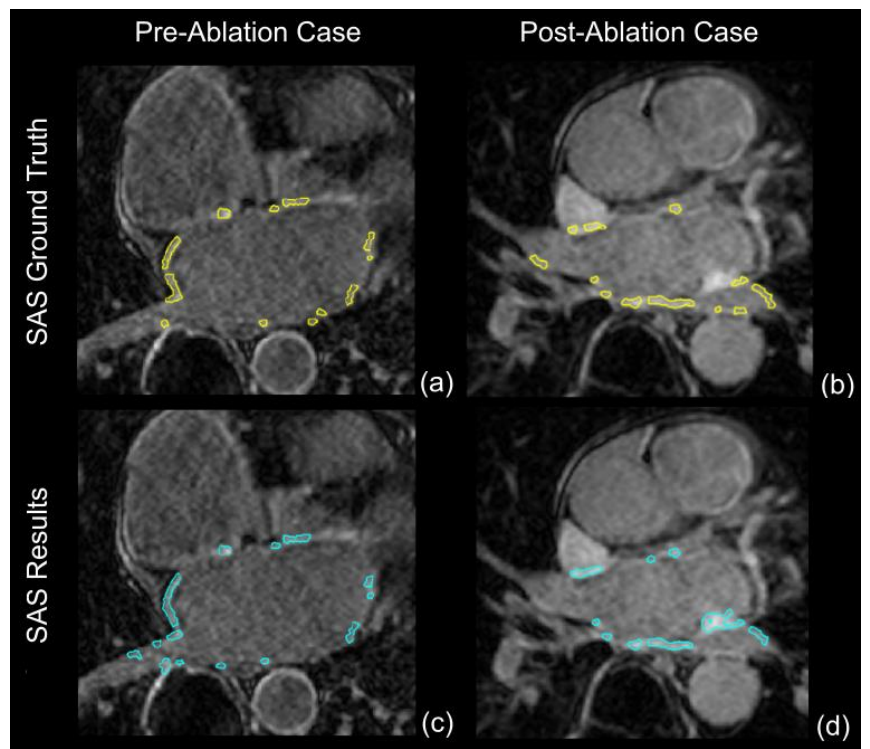

Figure 5: Final SAS results (cyan regions in (c) and (d)) for an example pre-ablation (left column) and an example post-ablation (right column) case compared to the ground truth (yellow regions in (a) and (b)).

As far as we know, this is the first study that applied a deep learning based classifier for fully automatic atrial scarring segmentation. The proposed framework has demonstrated a robust and efficient way to segment the atrial scarring from LGE MRI images. Based on the results on 20 longstanding persistent AF cases that contain both pre- and post-ablation LGE MRI scans, we believe that it is straightforward to deploy our method in the clinical environment. By using our framework, a patient-specific LA+PVs geometry model and an objective SAS can be achieved rapidly for individual AF patient without manual intervention. 


\section{REFERENCES}

A. Boldt, U. Wetzel, J. Lauschke, J. Weigl, J. Gummert, G. Hindricks, H. Kottkamp, and S. Dhein, "Fibrosis in left atrial tissue of patients with atrial fibrillation with and without underlying mitral valve disease.," Heart, vol. 90, no. 4, pp. 400-405, 2004.

[2] D. Ravanelli, E. C. Dal Piaz, M. Centonze, G. Casagranda, M. Marini, M. Del Greco, R. Karim, K. Rhode, and A. Valentini, “A novel skeleton based quantification and 3-D volumetric visualization of left atrium fibrosis using late gadolinium enhancement magnetic resonance imaging," IEEE Trans. Med. Imaging, vol. 33, no. 2, pp. 566-576, 2014

[3] M. Haïssaguerre, P. Jaïs, D. C. Shah, A. Takahashi, M. Hocini, G. Quiniou, S. Garrigue, A. Le Mouroux, P. Le Métayer, and J. Clémenty, "Spontaneous Initiation of Atrial Fibrillation by Ectopic Beats Originating in the Pulmonary Veins," N. Engl. J. Med., vol. 339, no. 10 pp. 659-666, Sep. 1998

[4] E. J. Schmidt, R. P. Mallozzi, A. Thiagalingam, G. Holmvang, A D’Avila, R. Guhde, R. Darrow, G. S. Slavin, M. M. Fung, J. Dando, L. Foley, C. L. Dumoulin, and V. Y. Reddy, "Electroanatomic mapping and radiofrequency ablation of porcine left atria and atrioventricular nodes using magnetic resonance catheter tracking.," Circ. Arrhythm. Electrophysiol., vol. 2, no. 6, pp. 695-704, Dec. 2009.

[5] P. Kellman and A. E. Arai, "Cardiac imaging techniques for physicians: Late enhancement," J. Magn. Reson. Imaging, vol. 36, no. 3, pp. 529 542, Sep. 2012.

[6] D. C. Peters, J. V Wylie, T. H. Hauser, K. V Kissinger, R. M. Botnar, V. Essebag, M. E. Josephson, and W. J. Manning, "Detection of pulmonary vein and left atrial scar after catheter ablation with three-dimensional navigator-gated delayed enhancement MR imaging: initial experience.," Radiology, vol. 243, no. 3, pp. 690-695, 2007.

[7] R. S. Oakes, T. J. Badger, E. G. Kholmovski, N. Akoum, N. S. Burgon E. N. Fish, J. J. E. Blauer, S. N. Rao, E. V. R. Dibella, N. M. Segerson, M. Daccarett, J. Windfelder, C. J. McGann, D. Parker, R. S. MacLeod, and N. F. Marrouche, "Detection and quantification of left atrial structural remodeling with delayed-enhancement magnetic resonance imaging in patients with atrial fibrillation," Circulation, vol. 119, no. 13, pp. 1758-1767, 2009

[8] D. Perry, A. Morris, N. Burgon, C. McGann, R. MacLeod, and J. Cates, "Automatic classification of scar tissue in late gadolinium enhancement cardiac MRI for the assessment of left-atrial wall injury after radiofrequency ablation," in In SPIE Medical Imaging, 2012, p. 83151D.

[9] R. Karim, R. J. Housden, M. Balasubramaniam, Z. Chen, D. Perry, A Uddin, Y. Al-Beyatti, E. Palkhi, P. Acheampong, S. Obom, A. Hennemuth, Y. Lu, W. Bai, W. Shi, Y. Gao, H.-O. Peitgen, P. Radau, R. Razavi, A. Tannenbaum, D. Rueckert, J. Cates, T. Schaeffter, D. Peters, R. MacLeod, and K. Rhode, "Evaluation of current algorithms for segmentation of scar tissue from late gadolinium enhancement cardiovascular magnetic resonance of the left atrium: an open-access grand challenge.," J. Cardiovasc. Magn. Reson., vol. 15, pp. 105-122, 2013.

[10] G. Yang, X. Zhuang, H. Khan, S. Haldar, E. Nyktari, L. Li, X. Ye, G. Slabaugh, T. Wong, R. Mohiaddin, J. Keegan, and D. Firmin, "Multi-Atlas Propagation Based Left Atrium Segmentation Coupled with Super-Voxel Based Pulmonary Veins Delineation in Late Gadolinium-Enhanced Cardiac MRI," in SPIE Medical Imaging, 2017. p. In Press.

[11] B. R. Knowles, D. Caulfield, M. Cooklin, C. A. Rinaldi, J. Gill, J. Bostock, R. Razavi, T. Schaeffter, and K. S. Rhode, "3-D visualization of acute RF ablation lesions using MRI for the simultaneous determination of the patterns of necrosis and edema," IEEE Trans. Biomed. Eng., vol. 57, no. 6, pp. 1467-1475, 2010.

[12] R. Karim, A. Arujuna, R. J. Housden, J. Gill, H. Cliffe, K. Matharu, C. a Rinaldi, M. O'Neill, D. Rueckert, R. Razavi, T. Schaeffter, and K. Rhode, "A Method to Standardize Quantification of Left Atrial Scar from Delayed-Enhancement MR Images," Transl. Eng. Heal. Med. IEEE J., vol. 2, pp. 1-15, 2014.

[13] Q. Tao, E. G. Ipek, R. Shahzad, F. F. Berendsen, S. Nazarian, and R. J van der Geest, "Fully automatic segmentation of left atrium and pulmonary veins in late gadolinium-enhanced MRI: Towards objective atrial scar assessment.," J. Magn. Reson. Imaging, vol. 44, no. 2, pp. 346-354, Jan. 2016

[14] C. J. McGann, E. G. Kholmovski, R. S. Oakes, J. J. E. Blauer, M. Daccarett, N. Segerson, K. J. Airey, N. Akoum, E. Fish, T. J. Badger, E. V. R. DiBella, D. Parker, R. S. MacLeod, and N. F. Marrouche, "New Magnetic Resonance Imaging-Based Method for Defining the Extent of Left Atrial Wall Injury After the Ablation of Atrial Fibrillation," J. Am.
Coll. Cardiol., vol. 52, no. 15, pp. 1263-1271, 2008.

[15] J. L. Harrison, C. Sohns, N. W. Linton, R. Karim, S. E. Williams, K. S. Rhode, J. Gill, M. Cooklin, C. a. Rinaldi, M. Wright, T. Schaeffter, R. S. Razavi, and M. D. O’Neill, "Repeat Left Atrial Catheter Ablation: Cardiac Magnetic Resonance Prediction of Endocardial Voltage and Gaps in Ablation Lesion Sets," Circ. Arrhythmia Electrophysiol., vol. 8, no. 2, pp. 270-278, Apr. 2015.

[16] D. C. Peters, J. V. Wylie, T. H. Hauser, R. Nezafat, Y. Han, J. J. Woo, J. Taclas, K. V. Kissinger, B. Goddu, M. E. Josephson, and W. J. Manning, "Recurrence of Atrial Fibrillation Correlates With the Extent of Post-Procedural Late Gadolinium Enhancement. A Pilot Study," JACC Cardiovasc. Imaging, vol. 2, no. 3, pp. 308-316, 2009.

[17] J. Keegan, P. Jhooti, S. V. Babu-Narayan, P. Drivas, S. Ernst, and D. N Firmin, "Improved respiratory efficiency of 3D late gadolinium enhancement imaging using the continuously adaptive windowing strategy (CLAWS)," Magn. Reson. Med., vol. 71, no. 3, pp. 1064-1074, 2014

[18] J. Keegan, P. Drivas, and D. N. Firmin, "Navigator artifact reduction in three-dimensional late gadolinium enhancement imaging of the atria," Magn. Reson. Med., vol. 785, pp. 779-785, 2013.

[19] X. Zhuang, W. Bai, J. Song, S. Zhan, X. Qian, W. Shi, Y. Lian, and D. Rueckert, "Multiatlas whole heart segmentation of CT data using conditional entropy for atlas ranking and selection.," Med. Phys., vol. 42, no. 7, pp. 3822-33, Jul. 2015.

[20] G. Yang, X. Ye, G. Slabaugh, J. Keegan, R. Mohiaddin, and D. Firmin, "Differentiation of Pre-Ablation and Post-Ablation Late Gadolinium-Enhanced Cardiac MRI Scans of Longstanding Persistent Atrial Fibrillation Patients," in SPIE Medical Imaging, 2017, p. In Press.

[21] C. Tobon-Gomez, A. Geers, J. Peters, J. Weese, K. Pinto, R. Karim, T. Schaeffter, R. Razavi, and K. Rhode, "Benchmark for algorithms segmenting the left atrium from 3D CT and MRI datasets.," IEEE Trans. Med. Imaging, vol. 34, no. 7, pp. 1460-1473, Feb. 2015.

[22] X. Zhuang, K. S. Rhode, R. S. Razavi, D. J. Hawkes, and S. Ourselin, "A registration-based propagation framework for automatic whole heart segmentation of cardiac MRI," IEEE Trans. Med. Imaging, vol. 29, no. 9, pp. 1612-1625, 2010.

[23] X. Zhuang and J. Shen, "Multi-scale patch and multi-modality atlases for whole heart segmentation of MRI," Med. Image Anal., vol. 31, pp. 77-87, Jul. 2016.

[24] P. Thévenaz and M. Unser, "Optimization of mutual information for multiresolution image registration.," IEEE Trans. Image Process., vol. 9, no. 12 , pp. 2083-99, 2000

[25] X. Zhuang, S. Arridge, D. J. Hawkes, and S. Ourselin, "A nonrigid registration framework using spatially encoded mutual information and free-form deformations.," IEEE Trans. Med. Imaging, vol. 30, no. 10, pp. 1819-28, Oct. 2011.

[26] R. Achanta, A. Shaji, K. Smith, and A. Lucchi, "SLIC superpixels compared to state-of-the-art superpixel methods," IEEE Trans. Pattern Anal. Mach. Intell., vol. 34, no. 11, pp. 2274-2281, 2012.

[27] Y. Bengio, "Learning Deep Architectures for AI," Found. Trends ${ }^{\circledR}$ Mach. Learn., vol. 2, no. 1, pp. 1-127, 2009.

[28] A. Ng, "Sparse autoencoder CS294A Lecture notes 72," 2011.

[29] Q. Lin, S. Ye, X. Huang, S. Li, M. Zhang, Y. Xue, and W.-S. Chen, "Classification of Epileptic EEG Signals with Stacked Sparse Autoencoder Based on Deep Learning," in Intelligent Computing Methodologies, 2016, pp. 802-810.

[30] X. Zhuang, "Challenges and methodologies of fully automatic whole heart segmentation: a review.," J. Healthc. Eng., vol. 4, no. 3, pp. 371408, 2013.

[31] D. P. Huttenlocher, G. A. Klanderman, and W. J. Rucklidge, "Comparing images using the Hausdorff distance," IEEE Trans. Pattern Anal. Mach. Intell., vol. 15, no. 9, pp. 850-863, 1993.

[32] G. Yang, F. Raschke, T. R. Barrick, and F. A. Howe, "Manifold Learning in MR Spectroscopy using Nonlinear Dimensionality Reduction and Unsupervised Clustering," Magn. Reson. Med., vol. 74, no. 3, pp. 868-878, 2015.

[33] G. Yang, T. L. Jones, T. R. Barrick, and F. A. Howe, "Discrimination between glioblastoma multiforme and solitary metastasis using morphological features derived from the $\mathrm{p}: \mathrm{q}$ tensor decomposition of diffusion tensor imaging.," NMR Biomed., vol. 27, no. 9, pp. 1103 1111, Sep. 2014

[34] M. Soltaninejad, G. Yang, T. Lambrou, N. Allinson, T. L. Jones, T. R. Barrick, F. A. Howe, and X. Ye, "Automated brain tumour detection and segmentation using superpixel-based extremely randomized trees in FLAIR MRI.," Int. J. Comput. Assist. Radiol. Surg., Sep. 2016. 